\title{
EDITORIAL
}

\section{The benefits of whole lung lavage in pulmonary alveolar proteinosis}

\author{
C. Morgan
}

Pulmonary alveolar proteinosis (PAP) is an interesting, rare and enigmatic disease, which was first described relatively recently in 1958 [1]. The affected individuals suffer a progressive accumulation of lipoproteins in the alveolar space, resulting in steadily worsening gas exchange, and culminating in death from hypoxia and or cor pulmonale. Even though the alveolar space is full of Periodic Acid Schiffstaining proteinaceous material, the actual lung tissue is practically normal, although a minor component of interstitial involvement is probably present in most cases. Three variants of the disease have been recognised: congenital, secondary and acquired (or idiopathic). It is the latter that is of most importance in terms of prevalence $(\sim 0.2$ cases per million of population) and response to treatment.

Successful treatment of PAP by therapeutic irrigation of the lungs was reported a few years after the disease was first described, and this led to massive whole lung lavage (WLL) [2], where, under general anaesthesia, the two lungs are separated by endobronchial intubation and one lung is ventilated while the other is flooded with warm saline to progressively wash out the alveolar space. Although there have been several refinements in this technique and developments in anaesthesia and peri-operative care, the basis of the treatment remains much the same [3-5].

It is not surprising that there have been a lot of interesting and informative review articles concerning PAP [6-8], and in the past $10 \mathrm{yrs}$ it has emerged that the aetiology of the acquired form of the disease may well be an auto-immune response [9]. A very high proportion of patients suffering from PAP have been shown to have high titres of an antigranulocyte-macrophage colony-stimulating factor (GM-CSF) antibody in plasma and the proposed disease mechanism is that interference with GM-CSF regulation of alveolar lipoprotein turnover results in the massive net accumulation of surfactant-like material in the alveolar space. Animal experiments in transgenic mice [10] have substantiated the aetiology and, in addition, potential therapeutic advances.

Successful moderation of experimental PAP in mice using GM-CSF was followed by preliminary studies of GM-CSF in humans with PAP $[11,12]$. This led some authors to refer to the traditional approach of therapeutic WLL as a "stop-gap" treatment until the cytokine modulation could be refined and results improved [13]. However, the success rate so far with GM-CSF is not sufficient to displace WLL as the treatment of choice. Approximately $50-70 \%$ of patients following GMCSF treatment protocols have obtained significant improvements in physiology, radiology and symptoms, which means that a significant percentage failed to benefit. Apart from the failure rate, the inconvenience of daily injections, the cost ( € 130-230 per dose assuming 5-10 $\mu \mathrm{gm} \cdot \mathrm{kg}^{-1} \cdot \mathrm{day}^{-1}$ in a $70 \mathrm{~kg}$ patient) and, recently, the security of supply of this treatment

Correspondence: C. Morgan, Consultant in Anaesthesia \& Critical Care Medicine, Royal Brompton Hospital, Sydney Street, London SW3 6NP, UK. Fax: 44 2073518521. E-mail: c.morgan@rbh.nthames.nhs.uk have to be considered. In addition, the potential toxicity of GM-CSF when used long term is not yet fully understood.

This context makes the work of BECCARIA et al. [14], published in the current issue of the European Respiratory Journal, all the more important. As stated above, a large proportion of publications about PAP are, by nature, review articles. The vast majority of the others over the past $10 \mathrm{yrs}$ have been related to the GM-CSF story in some measure. This has left little in the way of investigations of or around aspects of treatment by WLL, yet it would be reasonable to assume that progress is being made.

The article by BECCARIA et al. [14] describes the systematic treatment and follow-up of a series of 21 patients with idiopathic PAP treated by WLL in a single centre (Pavia, Italy) over a 12-yr period. This is a large series of patients and, therefore, an important addition to the literature. A lot of the features of the series are typical of all the other previously published clinical series, i.e. concentration of effort, numbers and experience in a major centre with patients travelling from all over that nation to the treatment centre; very low incidence/prevalence of PAP; affected group of PAP patients with typical age, smoking habit and sex ratio as compared to other series; extremely good therapeutic response to WLL; and some patients lost from long-term follow-up, in part related to distance from home to the PAP centre.

The authors of the article in this issue of the $E R J$ were able to overcome the logistic difficulties of long-term follow-up in an impressive 16 out of 21 patients, and, from the repetitive assessment of these patients, a significant and sustained improvement in forced vital capacity (FVC), arterial oxygen tension $\left(\mathrm{Pa}, \mathrm{O}_{2}\right)$, alveolar-arterial oxygen tension difference $\left(P \mathrm{Aa}, \mathrm{O}_{2}\right)$, carbon monoxide diffusing capacity of the lung $(D \mathrm{~L}, \mathrm{CO})$ and mean walking distance was seen after WLL. In addition, the variations in the time course of these improvements are demonstrated. For instance, FVC improves immediately and continues to improve to normal values at 1 yr. Conversely, DL,CO shows no immediate improvement but, thereafter, improves over the following 6 months but usually not to normal values. Oxygenation, as expressed as $P a, \mathrm{O}_{2}$ or $P A a, \mathrm{O}_{2}$, follows a similar pattern to $D \mathrm{~L}, \mathrm{CO}$. The duration of benefit in those patients who were able to be followed-up for an extended period (11 patients were followed up for $\geqslant 3$ yrs after WLL with median duration of follow-up of $5 \pm 2 \mathrm{yrs}$ ) was impressive, with $70 \%$ of patients free from recurrent manifestations of PAP $>3$ yrs after WLL. The authors rightly stress that this compares very favourably to the literature, where pooled series [6] suggest a much shorter duration of clinical benefit at 15 months and $<20 \%$ of longterm ( $>3 \mathrm{yrs}$ ) follow-up patients remaining free from PAP manifestations. However, these pooled series include patients from many centres over several decades and should not be used as the gold standard assessment of the value of WLL in the modern era. This is particularly important with regard to the alternative therapeutic option of treatment by GM-CSF. The Pavia experience has been published at a crucial time and 
demonstrates the following: 1) the clinical efficacy of WLL in the treatment of PAP is confirmed (again); 2) the duration of improvement is significant (or even permanent); and 3) the safety of the procedure in the hands of an experienced team is impeccable with no deaths and no incidences of major morbidity in the past 12 yrs.

BECCARIA et al. [14] stress the apparent permanence of response in those patients who could be followed up long term. They relate this apparent improvement compared to previously reported series to refinements in their particular technique. There are two potential issues with this line of reasoning: first, all the long-term follow-up patients have some residual physiological and clinical deficit at the latest point in their follow-up period, and this may actually be interpreted as an indication for further treatment by WLL; secondly, the authors attribute the duration of beneficial response following WLL to the completeness of treatment during WLL, that is they clearly believe that by using a prolonged and highly invasive variant of WLL to ensure that every gram of material is removed from both lungs during one WLL treatment episode, the patient enjoys a longer duration of benefit compared to less aggressive variants of WLL. There is no element of randomised comparison between the Pavia style of WLL and other variants of WLL, indeed how could there be? It is, therefore, dubious whether the authors can actually sustain their theory that their WLL technique is superior. Nevertheless, they have thrown down a challenge to other PAP treatment centres to evaluate the short-term and long-term outcome of their patients.

It is likely that cumulative results from the other centres in the world performing WLL for the treatment of PAP mirror the Pavia experience. This is certainly true of the past $18 \mathrm{yrs}$ of treatment of PAP at the Royal Brompton Hospital in London, where $>30$ patients have been treated by WLL for idiopathic PAP. All have obtained full remission of PAP, although after a variable period and total number of episodes of WLL. There have been no significant complications of the treatment and where long-term follow-up has been possible, all patients who achieved remission have subsequently remained in remission. It is likely that such results would not end up being published because there is little or no additional knowledge involved, journal editors may not be interested in this as compared to other related subject matter, and the physicians performing the treatments may not have the option of prolonged follow-up data. There is, thus, a danger that the newer treatment with GM-CSF will attract higher numbers of citations, greater clinical interest and premature adoption as treatment of first choice, even though results so far are inferior to the traditional treatment by WLL. Of course, the attraction of avoiding hospital admission, general anaesthesia, invasive interventions and recovery must be compelling. This would be particularly so if the patient and primary attending physician are remote from the nearest specialist centre offering treatment by WLL. The primary attending physician may not even know that there is a specialist centre offering the option of WLL. In such circumstances, the temptation may well be that it would be simpler to embark on a course of therapeutic GM-CSF using the example of insulin-dependent diabetes to justify imposing the ritual of daily injections on the patient. However, as mentioned above, the long-term toxicity of GM-CSF is not yet delineated and some areas of Europe suffer intermittent failure of supply of the drug. It is very expensive by any standards and, based on assumptions about dose level, weight of patient and local price variations, it could cost $€ 23,000-44,000$ for 6 months' treatment, based on a recently quoted price of GM-CSF [15]. There is a $\sim 50 \%$ response rate, although some patients have been treated for $>6$ months. The therapeutic failures presumably move on to
WLL. Hospital and other costs related to WLL are also difficult to estimate and may not be strictly comparable between different institutions, however, the estimated cost per episode of WLL at the Royal Brompton Hospital is $\sim € 4,500-5,000$ and the median number of WLL treatments required to achieve long-term remission is around four, therefore, the total cost has a median value of around $€ 20,000$. The financial estimates are a very rough guide in both situations but serve to illustrate that the therapeutic superiority of WLL, as demonstrated by BECCARIA et al. [14], is matched by more favourable costs compared to treatment by GM-CSF.

In conclusion, the therapeutic efficacy and safety of whole lung lavage for idiopathic pulmonary alveolar proteinosis is once again confirmed in a large and current series. It would be useful if there were a mechanism, over and above publications in journals, for those involved in the care of patients with pulmonary alveolar proteinosis to share knowledge and pool results. Pulmonary alveolar proteinosis should always be managed in a centre with experience and knowledge and which has a track record in safe and effective whole lung lavage. The role of granulocyte-macrophage colony-stimulating factor in the treatment of pulmonary alveolar proteinosis is yet to be established, but it may be a valuable alternative to whole lung lavage where whole lung lavage has failed or is not available. The future of treatment of pulmonary alveolar proteinosis is interesting, and there is a real prospect that improved understanding of cytokine regulation of alveolar metabolism may lead to specific alternatives to granulocyte-macrophage colony-stimulating factor and the eventual retirement of whole lung lavage as a therapeutic entity, but not for a considerable while yet!

\section{References}

1. Rosen SH, Castleman B, Liebow AA. Pulmonary alveolar proteinosis. N Engl J Med 1958; 258: 1123-1142.

2. Ramirez RJ, Schultz RB, Dutton RE. Pulmonary alveolar proteinosis: a new technique and rationale for treatment. Arch Intern Med 1963; 112: 419-431.

3. Selecky PA, Wasserman K, Benfield JR, Lippmann M. The clinical and physiological effect of whole-lung lavage in pulmonary alveolar proteinosis: a ten-year experience. Ann Thorac Surg 1977; 24: 451-461.

4. DuBois RM, McAllister WA, Branthwaite MA. Alveolar proteinosis: diagnosis and treatment over a 10 -year period. Thorax 1983; 381: 360-363.

5. Prackash UBS, Barham SS, Carpenter HA, Dines DE, Marsh HM. Pulmonary alveolar lipoproteinos: experience with 34 cases and a review. Mayo Clin Proc 1987; 62: 499 519 .

6. Seymour JF, Presneill JJ. Pulmonary alveolar proteinosis: progress in the first 44 years. Am J Respir Crit Care Med 2002; 166: 215-235.

7. Trapnell BC, Whitsett JA, Nakata K. Pulmonary alveolar proteinosis. N Eng J Med 2003; 349: 2527-2539.

8. Shah PL, Hansell D, Lawson PR, Reid KBM, Morgan C. Pulmonary alveolar proteinosis: clinical aspects and current concepts on pathogenesis. Thorax 2000; 55: 67-77.

9. Kitamura $\mathrm{T}$, Tanaka $\mathrm{N}$, Watanabe $\mathrm{J}$, et al. Idiopathic pulmonary alveolar proteinosis as an autoimmune disease with neutralizing antibody against granulocyte-macrophage colony stimulating factor. J Exp Med 1999; 190: 875-880.

10. Reed JA, Ikegami M, Cianciolo ER, et al. Aerosolized GM-CSF ameliorates pulmonary alveolar proteinosis in GM-CSF deficient mice. Am J Physiol 1999; 276: L556L563.

11. Seymour JF, Doyle IR, Nakata K, et al. Relationship of anti-GM-CSF antibody concentration, surfactant protein A 
and B levels, and serum LDH to pulmonary parameters and response to GM-CSF therapy in patients with idiopathic alveolar proteinosis. Thorax 2003; 58: 252-257.

12. Kavuru MS, Sullivan EJ, Piccin R, Thomassen MJ, Stoller JK. Exogenous granulocyte-macrophage colony-stimulating factor administration for pulmonary alveolar proteinosis. Am J Respir Crit Care Med 2000; 161: 1143-1148.
13. Kavuru MS, Popovich M. Therapeutic whole lung lavage a stop-gap therapy for alveolar proteinosis. Chest 2002; 122: 1123-1124.

14. Beccaria M, Luisetti M, Rodi G, et al. Long-term durable benefit after whole lung lavage in pulmonary alveolar proteinosis. Eur Respir J 2004; 23: 526-531.

15. British National Formulary. http://www.bnf.org/Index.htm 